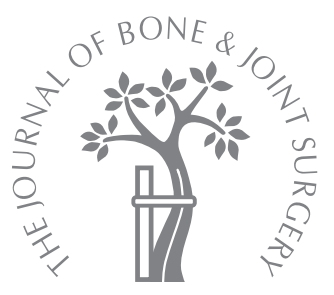

B-H. Hwang, J-Y. Yoon, C-H. Nam, K-A. Jung, S-C. Lee, C-D. Han, S-H. Moon

From Joint \& Arthritis Research, Himchan Hospital, Seoul, Korea

B-H. Hwang, MD, Orthopaedic Surgeon C-H. Nam, MD, PhD, Orthopaedic Surgeon

K-A. Jung, MD, Orthopaedic Surgeon

= S-C. Lee, Professor, MD, PhD Orthopaedic Surgeon

Himchan Hospital, Joint \& Arthritis Research, Department of Orthopaedic Surgery, 404-3 Mok-dong, Yangcheon-gu, Seoul 158-806, Korea.

In-Y. Yoon, MD, Physician Himchan Hospital, Joint \& Arthritis Research, Division of Rheumatology, 404-3 Mokdong, Yangcheon-gu, Seoul 158-806, Korea.

- C-D. Han, MD, PhD, Professor = $\mathrm{S}-\mathrm{H}$. Moon, MD, PhD, Professor

Yonsei University College of Medicine, Department of Orthopaedic Surgery, 250 Seongsanno, Seodaemun-gu, Seoul 120-752, Korea.

Correspondence should be sent to Dr S-C. Lee; e-mail: scleeos@gmail.com

(C)2012 British Editorial Society of Bone and Joint Surgery doi:10.1302/0301-620X.94B5 $28125 \$ 2.00$

$J$ Bone Joint Surg Br 2012;94-B:656-9.

Received 9 August 2011;

Accepted after revision

5 January 2012

\title{
Fungal peri-prosthetic joint infection after primary total knee replacement
}

\begin{abstract}
We retrospectively reviewed 30 two-stage revision procedures in 28 patients performed for fungal peri-prosthetic joint infection (PJI) after a primary total knee replacement. Patients were followed for at least two years or until the infection recurred. The mean follow-up for patients who remained free of infection was 4.3 years (2.3 to 6.1). Overall, 17 patients were assessed as American Society of Anesthesiologists grade 3 or 4 . The surgical protocol included removal of the infected implant, vigorous debridement and insertion of an articulating cement spacer. This was followed by at least six weeks of antimicrobial treatment and delayed reimplantation in all patients. The mean interval between removal of the prosthesis and reimplantation was 9.5 weeks ( 6 to 24). After reimplantation, patients took antifungal agents orally for a maximum of six months. Two knees became reinfected at one and two months post-operatively, respectively: one of these subsequently required arthrodesis because of uncontrolled infection.

Fungal PJls can be treated successfully by removal of all infected material, appropriate antimicrobial treatment and delayed reimplantation.
\end{abstract}

Peri-prosthetic joint infection (PJI) is one of the most serious complications of total knee replacement (TKR). ${ }^{1}$ Its incidence has increased in recent years, but fungal PJIs are rarely reported. ${ }^{2-12}$ These are generally indolent, associated with impaired host immunity, and may be indistinguishable from common bacterial infections. Their diagnosis and treatment are therefore often delayed, which may prejudice the outcome.

Fungal PJIs pose a number of problems, both surgically and medically. Although twostage reimplantation gives the greatest likelihood of a good outcome in common bacterial PJI, only selected patients have undergone reimplantation following fungal PJIs. $4,6,8,13,14$

The aim of this study was to identify the clinical features of fungal infection of a TKR and the outcome of two-stage reimplantation. We hypothesised that an infected TKR could be salvaged successfully in most patients by removal of all the infected components, appropriate antimicrobial treatment and interval reimplantation.

\section{Patients and Methods}

After Institutional Review Board approval, we performed a retrospective study using data collected from the arthroplasty databases in our four centres, where 25585 primary TKRs were undertaken between 2005 and 2009.
These included 30 patients (32 knees) with fungal PJIs. Of these, two patients (two knees) were lost to follow-up. Thus, a total of 28 patients (30 knees) were available for clinical and radiological assessment at a minimum of two years or until infection recurred. Their mean age at the time of diagnosis was 69 years (49 to 83). The mean follow-up for 26 patients (28 knees) who remained free of infection was 4.3 years (2.3 to 6.1$)$.

Deep PJI was confirmed if at least three of the following criteria were met: 1) a C-reactive protein level $(\mathrm{CRP})>1 \mathrm{mg} / \mathrm{dl} ; 2)$ an erythrocyte sedimentation rate $(\mathrm{ESR})>30 \mathrm{~mm} / \mathrm{h} ; 3)$ a positive culture of joint aspirate; 4) the appearance of pus at operation; and 5) a positive intra-operative culture. ${ }^{15}$ During the period of the study, surgeons from each centre obtained multiple pre-operative and intra-operative cultures for fungi, acid-fast bacilli and bacteria from the infected knees. The fungi were cultured on Sabouraud dextrose agar and then incubated for four weeks at $24^{\circ} \mathrm{C}$ to $25^{\circ} \mathrm{C}$.

Two-stage reimplantation consisted of removal of all prosthetic components, insertion of a vancomycin-impregnated cement ( $2 \mathrm{~g}$ vancomycin per $40 \mathrm{~g}$ cement) articulating spacer, and delayed reimplantation. After the first stage, patients underwent physiotherapy to encourage passive knee movement and preserve quadriceps strength. Delayed reimplantation 
was performed when the wound was healthy and the patient was clinically stable with normal CRP.

Clinical and radiological data of all patients undergoing joint replacement are collected prospectively by the joint registries of our institutions. Patients were examined before surgery and at six weeks, six months and one year after surgery, and yearly thereafter. The Knee Society clinical score $(\mathrm{KSS})^{16}$ and Western Ontario and McMaster Universities Osteoarthritis Index score (WOMAC) ${ }^{17}$ were obtained. In order to assess potential immunological comorbidities, an anaesthetist and physicians at each centre assessed the patient's American Society of Anesthesiologists (ASA) ${ }^{18}$ grade pre-operatively. Patients were subdivided according to their ASA grade. The low-risk subgroup (ASA grades 1 and 2) included patients who had no comorbidities or who had a mild illness that did not affect their daily life. The high-risk subgroup (ASA grades 3 and 4) included patients who had moderate or severe illness which affected daily life. Patient satisfaction was assessed by asking 'Are you satisfied with the results of your reimplanted prosthesis?' Response categories consisted of very satisfied, somewhat satisfied, somewhat dissatisfied, and very dissatisfied. All patients were also followed up in a telephone survey with the patients themselves and with their first-degree relatives. Fluoroscopically assisted anteroposterior (AP) and lateral radiographs with a skyline view of the patella were obtained according to the method of the Knee Society to determine the presence of radiolucent lines (RLLs) at the bone-cement interface and any osteolysis. ${ }^{19}$ All data were collected and analysed by an author (BHH) who was not involved in any of the index operations.

\section{Results}

The mean interval between TKR and the diagnosis of PJI was 19.6 months ( 2 to 51 ). In all, 17 patients belonged to the high-risk ASA groups. At diagnosis, four patients were taking systemic corticosteroids, two for rheumatoid arthritis and two for adrenal insufficiency. Two patients had a past history of fungal arthritis of the knee before their primary TKR. Their knees had been infected with Candida albicans and Pichia anomala, respectively, and treated by arthroscopic debridement and antifungal therapy 18 months and 11 months before TKR, respectively. No patient had a past history of bacterial infection of the knee. Laboratory findings and microbiology. At the diagnosis of PJI, the mean white cell count was 5300 cells $/ \mu$ l (3100 to 9600), ESR $39 \mathrm{~mm} / \mathrm{h}$ (11 to 102), and CRP $4.3 \mathrm{mg} / \mathrm{dl}$ (0.8 to 13.2). Pre-operative analysis of the joint fluid revealed a mean synovial white cell count of 5072 cells/ $\mu 1$ (680 to $14620)$, and neutrophils comprised a mean of $65 \%(16 \%$ to $97 \%$ ). Laboratory examinations, such as blood or urine culture, were done to exclude systemic infection and none was found.

Most fungal PJIs were caused by Candida species (24 knees, 80\%), of which Candida parapsilosis was the most common (12 knees, 50\%). Definitive fungal organisms were grown from 21 knees following aspiration and, of these, 17 gave the same result from intra-operative culture. Coexisting bacteria were isolated in six knees, four of which initially gave a positive bacterial culture before the fungal pathogen was isolated four weeks later. Bilateral fungal PJIs occurred in two patients, both of whom were ASA grade 3: one had a history of diabetes mellitus, hypertension and an arrhythmia, while the other had a history of repeated systemic steroid use, diabetes mellitus and angina. Clinical and radiological features. Most patients had the symptoms and signs of an infected knee, including swelling, pain or limitation of movement. Draining sinuses were present in seven knees. In three knees, peri-prosthetic RLLs or osteolysis preceded these signs and symptoms. None had fever, weight loss, or anorexia; 12 knees showed evidence of progressive RLLs, extensive osteolysis or loosening of the prosthetic components.

Surgical and medical treatments. Debridement with retention of the prosthesis was the initial surgical procedure in four knees (four patients), but none of these resulted in resolution of the infection and each patient subsequently required removal of the components, debridement and insertion of a cement spacer. Meanwhile, the remaining 26 knees were treated with resection arthroplasty from the outset. Two knees needed a further debridement and exchange of the cement spacer because of uncontrolled infection. All knees were followed by delayed reimplantation at a mean of 9.5 weeks (6 to 24) after resection arthroplasty, and intra-operative cultures were negative.

After their initial surgical treatment (resection arthroplasty or debridement), patients were treated with at least six weeks of antifungal agents based on the specific species of fungus grown. Amphotericin $\mathrm{B}$ was given to 24 patients, followed in three of these by fluconazole. Fluconazole was initially given to four patients. After reimplantation, oral fluconazole was prescribed for a maximum of six months, accompanied by regular monitoring of liver function. However, six patients developed adverse events related to their antifungal therapy. The serum liver enzymes were elevated in two patients, one patient developed acute renal failure and a further patient an amphotericin B neuropathy. Significant gastrointestinal disturbance secondary to the use of fluconazole was seen in the other patients.

Treatment outcomes and recurrent infections. Two knees had recurrent infection after successful reimplantation: one had continued drainage from the wound two months postoperatively and underwent further debridement and longterm suppressive antimicrobial therapy despite identifying no infecting organism. The other, from which Staphylococcus epidermidis was grown one month post-operatively, also underwent further debridement, but eventually required arthrodesis of the knee because of uncontrolled infection. Radiological evidence of fusion was seen two years later. Both knees were free of infection at the most recent follow-up. 
At follow-up, excluding the two knees with recurrent infection, the mean KSS pain score was 77 (40 to 95), the mean KSS function score was 69 (35 to 90), and the mean WOMAC score was 71 (36 to 91). The mean active nonweight-bearing range of movement was from $2.5^{\circ}\left(0^{\circ}\right.$ to $\left.15^{\circ}\right)$ to $105^{\circ}\left(65^{\circ}\right.$ to $\left.125^{\circ}\right)$. Most patients performed activities of daily living well, but three were dependent on another person. Overall, 20 patients ( 20 knees, $71 \%$ ) were satisfied with the revision TKR. There was no radiological evidence of loosening of the revised prosthesis in any patient.

\section{Discussion}

Fungal peri-prosthetic joint infections are rare. In most previous studies C. albicans has been the principal responsible organism, but in this study C. parapsilosis was found to be the principal cause., ${ }^{3,7-9}$ This incidence may be on the rise because of the increasing number of TKRs being carried out in patients with diminished host immunity for any reason. . $^{2-4,8,10,12,13,20}$ The possibility of a fungal infection should be considered in such patients with evidence of periprosthetic infection or loosening. In this study most patients had identifiable predisposing factors for such infections.

The onset of symptoms is subtle. Most present weeks to years after TKR. In our study this mean time was 19.6 months, which is similar to that previously reported. ${ }^{2}$ Low-grade pain and swelling are the most common features, but the only signs may be progressive peri-prosthetic radiolucency or osteolysis, ${ }^{5}$ which occurred in three knees in this study. Sinus tracks may also be present owing to the indolent nature of the infection. The diagnosis may be delayed because of difficulty in identifying the organism, or because the possibility of fungal infection has not been considered. Laboratory tests could not distinguish these PJIs from a bacterial infection. These results were consistent with previous findings. ${ }^{13}$

In this study, other bacteria were initially isolated in four knees before fungal pathogens were cultured, which complicated the diagnosis. The clinical presentation was the same as that of a standard bacterial peri-prosthetic infection. In cases where symptoms persist with negative aerobic and anaerobic cultures, diagnosis is dependent on a high level of clinical suspicion. Multiple specimens are needed for a wide variety of tests.

The preferred treatment of a PJI caused by common pathogens is two-stage reimplantation. ${ }^{21}$ By contrast, various treatments have been described for fungal PJIs. The success of single-stage exchange has only been reported in one case. ${ }^{11}$ Although several reports ${ }^{2,7,9,10}$ have described the successful treatment of fungal PJI with retention of the prosthesis, these infections were treated in the acute stage. When the infection is chronic, most authors prefer to remove all the infected material according to the guidelines of the Infectious Diseases Society of America (IDSA). ${ }^{20}$ Resolution of a fungal infection without removal of the prosthesis is unlikely, as fungal infection develops mainly as a chronic condition in an immunocompromised patient. ${ }^{22,23}$
Azzam et $\mathrm{al}^{13}$ reported that two-stage reimplantation was the treatment of choice and gave good results in a multicentre study of 31 patients with a fungal PJI. In our study, although two knees became reinfected, most patients did well after a two-stage reimplantation, and 20 patients (20 knees; 71\%) were satisfied with their revision.

The articulating cement spacer allows patients to retain extensor function and knee movement, and probably improves the functional outcome. However, there is no consensus about the type and dose of antifungal agents that can be mixed with cement. Although amphotericin B, which is heat stable and available in powder form, appears to be an effective agent, previous studies have shown that it is not delivered in a sufficiently high local dose when mixed with cement. ${ }^{24-26}$ In our study, vancomycin-impregnated cement was used in all patients.

It is believed that a longer interval between removal of components and reimplantation is required for fungal infections. Although this may lessen the risk of recurrent infection, it also increases the risk of morbidity, including a stiff or dysfunctional knee. In this study, patients with fungal PJI underwent delayed reimplantation after a mean of 9.5 weeks. One study of successful reimplantation for fungal PJI caused by Candida showed that the interval could be shortened to three months. ${ }^{6}$ We agree that this is possible, if eradication of infection can be confirmed by negative repeat cultures and the inflammatory markers have returned to normal levels, especially if it has been possible to improve the patient's host immunity before reimplantation.

In most previously reported cases, amphotericin B and fluconazole have been shown to be effective, ${ }^{7,20}$ although resistance of Candida species to azole drugs has been reported. ${ }^{11}$ There is evidence that oral fluconazole penetrates tissues well and has excellent bioavailability. ${ }^{27} \mathrm{How}-$ ever, the required duration of treatment is unclear. On the basis of previous reports, ${ }^{10,12}$ six to 12 months of treatment appear to be needed. Recent guidelines from the Infectious Diseases Society of America ${ }^{20}$ recommend resection arthroplasty with a minimum six-week course of an antifungal agent, which mirrors the recommendation for native joint infection. Phelan et $\mathrm{al}^{8}$ noted a recurrence rate of $20 \%$ after two-stage reimplantation for the treatment of candidal PJI. In our study, all except two knees showed no evidence of residual chronic infection at the time of the most recent follow-up. These findings suggest that at least six weeks of antifungal agents are needed before reimplantation, followed by oral fluconazole therapy for a maximum of six months. A multidisciplinary approach involving infectious disease specialists and clinical pharmacologists is essential for the selection of appropriate antimicrobial agents and to reduce the interval to surgery.

Bilateral PJIs caused by any pathogen are very uncommon, ${ }^{28,29}$ and we found no reports in the literature of fungal PJI involving bilateral TKRs. However, we saw this in two patients who underwent staged bilateral TKR three and four years before presentation. Both patients were ASA 
grade 3 and were poorly nourished at the time of operation. Although their infection did not recur, they had a poor outcome and were dissatisfied with the procedure.

The main limitations of this study include the inherent problems associated with a non-randomised, retrospective study, such as recall bias. The number of patients was also small, which weakens the power of the data, and it did not include a susceptibility test for antifungal agent for every patient. Unfortunately, different techniques of fungal culture exist with variation in culture media, transport and volume required. Clinicians should be aware that interpretation may vary with the test being used. ${ }^{30}$ An understanding of the pharmacokinetic properties and minimal inhibitory concentrations of antifungal agents is essential when deciding on the appropriate medical treatment. In our study, patients were treated with a regimen based on the species of fungus isolated from culture and the laboratory results. Our study does not give complete treatment guidelines for each type of fungal PJI because of our limited experience and the short follow-up.

In conclusion, the findings of this study support the hypothesis that two-stage revision TKR with appropriate antimicrobial treatment can give good results in most patients with a fungal PJI. It should be considered in patients with negative cultures or if host immunity is compromised. Obtaining the appropriate cultures and timing of reimplantation are essential for a successful outcome. Correction of nutritional and laboratory status should also be made in order to optimise host immunity before and after treatment of this group of patients.

\section{Supplementary material}

$\ddot{e}$ Three tables, detailing i) the clinical and microbiological features of the 30 total knee replacements, ii) the potential immunity-impairing factors in the 28 patients, and iii) the treatment of the 30 knees, are available with the electronic version of this article on our website www.jbjs.boneandjoint.org.uk

No benefits in any form have been received or will be received from a commercial party related directly or indirectly to the subject of this article.

\section{References}

1. Kurtz SM, Lau E, Schmier J, et al. Infection burden for hip and knee arthroplasty in the United States. J Arthroplasty 2008;23:984-991.

2. Brooks DH, Pupparo F. Successful salvage of a primary total knee arthroplasty infected with Candida parapsilosis. J Arthroplasty 1998;13:707-712.

3. Cardinal E, Braunstein EM, Capello WN, Heck DA. Candida albicans infection of prosthetic joints. Orthopedics 1996;19:247-251.

4. Hennessy MJ. Infection of a total knee arthroplasty by Candida parapsilosis: a case report of successful treatment by joint reimplantation with a literature review. $\mathrm{Am} \mathrm{J}$ Knee Surg 1996:9:133-136.
5. Darouiche RO, Hamill RJ, Musher DM, Young EJ, Harris RL. Periprosthetic candidal infections following arthroplasty. Rev Infect Dis 1989;11:89-96.

6. Yang SH, Pao JL, Hang YS. Staged reimplantation of total knee arthroplasty after Candida infection. J Arthroplasty 2001;16:529-532.

7. Merrer J, Dupont B, Nieszkowska A, De Jonghe B, Outin H. Candida albicans prosthetic arthritis treated with fluconazole alone. J Infect 2001;42:208-209.

8. Phelan DM, Osmon DR, Keating MR, Hanssen AD. Delayed reimplantation arthroplasty for candidal prosthetic joint infection: a report of 4 cases and review of the literature. Clin Infect Dis 2002;34:930-938.

9. Simonian PT, Brause BD, Wickiewicz TL. Candida infection after total knee arthroplasty: management without resection or amphotericin B. J Arthroplasty 1997;12:825-829.

10. Fukasawa N, Shirakura K. Candida arthritis after total knee arthroplasty: a case of successful treatment without prosthesis removal. Acta Orthop Scand 1997:68:306307

11. Selmon GP, Slater RN, Shepperd JA, Wright EP. Successful 1-stage exchange total knee arthroplasty for fungal infection. J Arthroplasty 1998;13:114-115.

12. Tunkel AR, Thomas CY, Wispelwey B. Candida prosthetic arthritis: report of a case treated with fluconazole and review of the literature. Am J Med 1993:94:100103

13. Azzam K, Parvizi J, Jungkind D, et al. Microbiological, clinical, and surgical features of fungal prosthetic joint infections: a multi-institutional experience. J Bone Joint Surg [Am]2009;91-A:142-149.

14. Marculescu CE, Berbari EF, Cockerill FR 3rd, Osmon DR. Fungi, mycobacteria, zoonotic and other organisms in prosthetic joint infection. Clin Orthop 2006;451:6472.

15. Parvizi J, Ghanem E, Menashe S, Barrack RL, Bauer TW. Periprosthetic infection: what are the diagnostic challenges? J Bone Joint Surg [Am]2006;88-A:138-147.

16. Insall JN, Dorr LD, Scott RD, Scott WN. Rationale of the Knee Society clinical rating system. Clin Orthop 1989;248:13-14.

17. Bellamy N. WOMAC osteoarthritis index: a user's guide IX. Brisbane: The University of Queensland, 2008:176.

18. Dripps RD, Lamont A, Eckenhoff JE. The role of anesthesia in surgical mortality. JAMA 1961;178:261-266.

19. Ewald FC. The Knee Society total knee arthroplasty roentgenographic evaluation and scoring system. Clin Orthop 1989;248:9-12.

20. Pappas PG, Kauffman CA, Andes D, et al. Clinical practice guidelines for the management of candidiasis: 2009 update by the Infectious Diseases Society of America. Clin Infect Dis 2009;48:503-535

21. Goldman RT, Scuderi GR, Insall JN. 2-stage reimplantation for infected total knee replacement. Clin Orthop 1996;331:118-124.

22. Silva M, Tharani R, Schmalzried TP. Results of direct exchange or debridement of the infected total knee arthroplasty. Clin Orthop 2002;404:125-131.

23. Crockarell JR, Hanssen AD, Osmon DR, Morrey BF. Treatment of infection with debridement and retention of the components following hip arthroplasty. J Bone Joint Surg [Am] 1998;80-A:1306-1313.

24. Kuhn DM, Chandra J, Mukheriee PK, Ghannoum MA. Comparison of biofilms formed by Candida albicans and Candida parapsilosis on bioprosthetic surfaces. Infect Immun 2002;70:878-888.

25. Goss B, Lutton C, Weinrauch P, et al. Elution and mechanical properties of antifungal bone cement. J Arthroplasty 2007;22:902-908.

26. Marra F, Robbins GM, Masri BA, et al. Amphotericin B-loaded bone cement to treat osteomyelitis caused by Candida albicans. Can J Surg 2001;44:383-386.

27. Brammer KW, Farrow PR, Faulkner JK. Pharmacokinetics and tissue penetration of fluconazole in humans. Rev Infect Dis 1990;12(Suppl 3):318-326.

28. Porat MD, Austin MS. Bilateral knee periprosthetic infection with Mycobacterium fortuitum. J Arthroplasty 2008;23:787-789.

29. Kobayashi H, Hall GS, Tuohy MJ, et al. Bilateral periprosthetic joint infection caused by Salmonella enterica serotype Enteritidis, and identification of Salmonella sp using molecular techniques. Int J Infect Dis 2009;13:463-466.

30. Rodloff C, Koch D, Schaumann R. Epidemiology and antifungal resistance in invasive candidiasis. Eur J Med Res 2011;16:187-195. 